\title{
Letter to Editor in Response to the Article Entitled "Surgical Methods and Experiences of Surgeons Did Not Significantly Affect the Recovery in Phonation Following Reconstruction of the Recurrent Laryngeal Nerve"
}

\author{
Chandan Kumar Jha ${ }^{1}$ - Anjali Mishra ${ }^{1}$
}

Published online: 19 September 2016

(C) Société Internationale de Chirurgie 2016

\section{Dear Sir,}

We read with interest the article entitled "Surgical Methods and Experiences of Surgeons did not Significantly Affect the Recovery in Phonation Following Reconstruction of the Recurrent Laryngeal Nerve (RLN)" by $\mathrm{K}$. Yoshioka et al. published in World Journal of Surgery [1]. When it comes to the subject of voice and RLN resection and/or anastomosis, any article from Dr. Miyauchi's group becomes a must read for all thyroid surgeons and it was the same again. The authors have shown that recovery of voice following resection and immediate reconstruction of RLN was not associated with gender, age, preoperative VCP, surgical method of reconstruction, or experience of the surgeon. At our institute we often operate on locally advanced thyroid carcinoma patients [2]. Some of these patients have gross invasion of RLN, which many times have to be resected in order to avoid leaving gross disease. We do not follow a policy of routine anastomosis, especially when a segment of nerve is resected. We have observed that the voice is generally well compensated in few weeks. Although we have not done a formal study on this subject, more often than not, patient's subjective assessment of their own voice does not seem to differ in group of patients who had undergone anastomosis compared to those who have not had anastomosis following RLN resections. It brings us to the question that what was the correlation of the findings of maximum phonation time

Anjali Mishra

anjali@sgpgi.ac.in; anjali_mishra2000@yahoo.com

1 Department of Endocrine Surgery, Sanjay Gandhi Postgraduate Institute of Medical Sciences, Raebareli Road, Lucknow 226 014, India
(MPT) and mean airflow rate (MFR) with the patient's own assessment of quality of voice? Another query that we would like to make is that whether some or all of these patients underwent any voice therapy after nerve anastomosis?

Voice is not just the function of RLN integrity, a host of other factors may also affect it [3-5], division of strap muscles, status of the external branch of superior laryngeal nerve being some of those which are important in case of thyroidectomy. And, this is why we felt that it would have been better if the control group could have been selected from the patients operated by the authors' group who had been subjected to a uniform technique of thyroidectomy rather than those operated by other surgeons.

\section{References}

1. Yoshioka K, Miyauchi A, Fukushima M, Kobayashi K, Kihara M, Miya A (2016) Surgical methods and experiences of surgeons did not significantly affect the recovery in phonation following reconstruction of the recurrent laryngeal nerve. World J Surg. doi:10.1007/s00268-016-3634-7

2. Bhargav PR, Mishra A, Agarwal G, Agarwal A, Pradhan PK, Gambhir S, Verma AK, Mishra SK (2010) Long-term outcome of differentiated thyroid carcinoma: experience in a developing country. World J Surg 34:40-47. doi:10.1007/s00268-009-0293-y

3. Moris D, Mantonakis E, Makris M, Michalinos A, Vernadakis S (2014) Hoarseness after thyroidectomy: blame the endocrine surgeon alone? Hormones (Athens) 13:5-15

4. Hong KH, Yang YS, Lee HD, Yoon YS, Hong YT (2015) The effect of total thyroidectomy on the speech production. Clin Exp Otorhinolaryngol 8:155-160 (Epub 2015 May 13)

5. Roubeau B, Chevrie-Muller C, Guily JLS (1997) Electromyographic activity of strap and cricothyroid muscles in pitch change. Acta Oto-Laryngol 117:459-464 